\title{
A Universal Relation between Electron Density Minima and Ionic Radii in Ceramics
}

\author{
Masahito Yoshino ${ }^{1}$, Masahiko Morinaga ${ }^{1}$, Akihiro Shimode ${ }^{1, *}$, \\ Kousuke Okabayashi ${ }^{1, *}$, Hirohide Nakamatsu ${ }^{2}$ and Rika Sekine ${ }^{3}$ \\ ${ }^{1}$ Department of Materials Science and Engineering, Graduate School of Engineering, Nagoya University, Nagoya 464-8603, Japan \\ ${ }^{2}$ Insitute for Chemical Research, Kyoto University, Uji 611-0011, Japan \\ ${ }^{3}$ Department of Chemistry, Faculty of Science, Shizuoka University, Hamamatsu 422-8529, Japan
}

\begin{abstract}
A universal relation between electron density minima and ionic radii, was discovered from the first principles calculations of electronic structures in ceramics over 60 species, including oxides, borides, carbides, nitride and fluorides. Every ceramic falls on a curve of $\log \left(\rho_{\text {min }} Z^{-3}\right)$ vs. $2(Z / n) r_{\min }$, where $\rho_{\min }$ is the minimum electron density in the line linking the first-nearest-neighbor nuclei, $r_{\text {min }}$ is the distance $r$ at $\rho_{\min }, Z$ is the atomic number, and $n$ is the principal quantum number.
\end{abstract}

(Received January 21, 2004; Accepted March 22, 2004)

Keywords: electron density, ceramics, oxides, borides, carbides, nitrides, fluorides, molecular orbital method, ionic radius

\section{Introduction}

Knowledge of spatial electron density distribution, $\rho(r)$, is so fundamental for the basic understanding of the nature of the chemical bond between atoms in materials. For convenience, several methods have been proposed for analyzing $\rho(r)$. For example, following the Mulliken population analysis method, ${ }^{1,2)}$ overlap populations and orbital populations are calculated and used as a measure of chemical interactions between atoms. Such interactions may be also characterized by the Laplacian of the electron density, $\nabla^{2} \rho(r) .{ }^{3)}$ However, despite great efforts, there has been little information of the feature of $\rho(r)$ common to every ceramic material.

Presented in this paper is a universal relation between electron density minima and ionic radii, which was discovered from the DV-X $\alpha$ molecular orbital calculations of electronic structures in a variety of ceramics.

\section{DV-X $\alpha$ Molecular Orbital Method and Cluster Models}

In the DV-X $\alpha$ molecular orbital method, ${ }^{4-7)}$ the Slater's $\mathrm{X} \alpha$ potential is adopted to represent the exchange-correlation interaction between electrons. For the calculation a large-size cluster model was constructed using experimental data on the crystal structure of ceramics. For example, it was a $\mathrm{Mg}_{32} \mathrm{O}_{32}$ cluster for $\mathrm{MgO}$ with the rock salt structure, and a $\mathrm{Ti}_{7} \mathrm{Ba}_{8} \mathrm{O}_{36}$ cluster for $\mathrm{BaTiO}_{3}$ with the perovskite-type structure.

In order to take account of the Madelung potential between ions, point charges were arranged around the cluster. For example, in case of perovskite-type oxides, a space with the size about $8 \mathrm{~nm} \times 8 \mathrm{~nm} \times 8 \mathrm{~nm}$ was set around the cluster and point charges were arranged at every position of ions inside this space. However, in case of $\mathrm{NaCl}$-type ceramics any point charges were not arranged around the cluster, and instead, as shown in Fig. 1, the atoms located in the central part of the cluster were surrounded completely by a number

*Graduate Student, Nagoya University

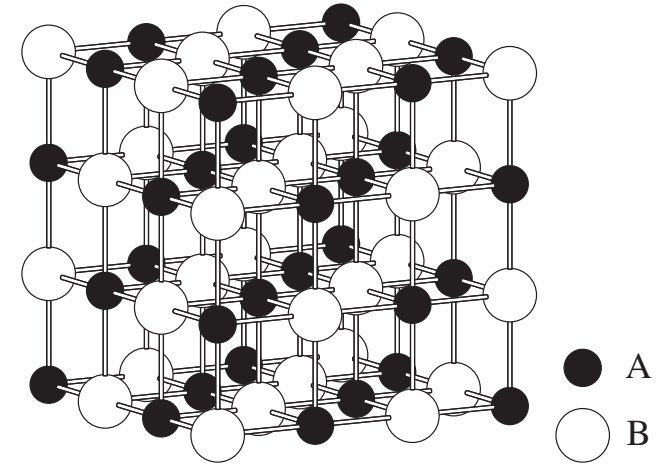

Fig. 1 Cluster model used in the calculations for NaCl-type ceramics, AB.

of neighboring atoms. Because, it was difficult to define the point charge state for some ceramics with covalent character such as $\mathrm{ZrB}$ and $\mathrm{TiC}$. But, even in case of $\mathrm{MgO}$ with ionic character, the calculated electron density with this cluster was found to be comparable to the result obtained by the standard scheme of placing point charges around the cluster. Therefore, in any cases the calculations without using a Madelung potential in the $\mathrm{NaCl}$-type ceramics could be verified for the purpose of obtaining electron density distributions accurately in them.

\section{Results and Discussion}

\subsection{Electron density in metal oxides}

\subsubsection{Natural logarithm plots of electron density}

One example of the calculated results of electron density, $\rho(r)$, is shown in Fig. 2 for $\mathrm{MgO}$. The natural logarithm of the electron density, $\log \rho(r)$, is illustrated along the line linking the first-nearest neighbor $\mathrm{Mg}$ and $\mathrm{O}$ nuclei. Here, the logarithm was taken to detect a very small change in the $\rho(r)$ curve. As is evident from Fig. 2, $\log \rho(r)$ decreases monotonously with the distance, $r$, from the oxygen nucleus, but the curve is separated into two regions in the oxygen side, and each region has own slope. The slope varies with the 


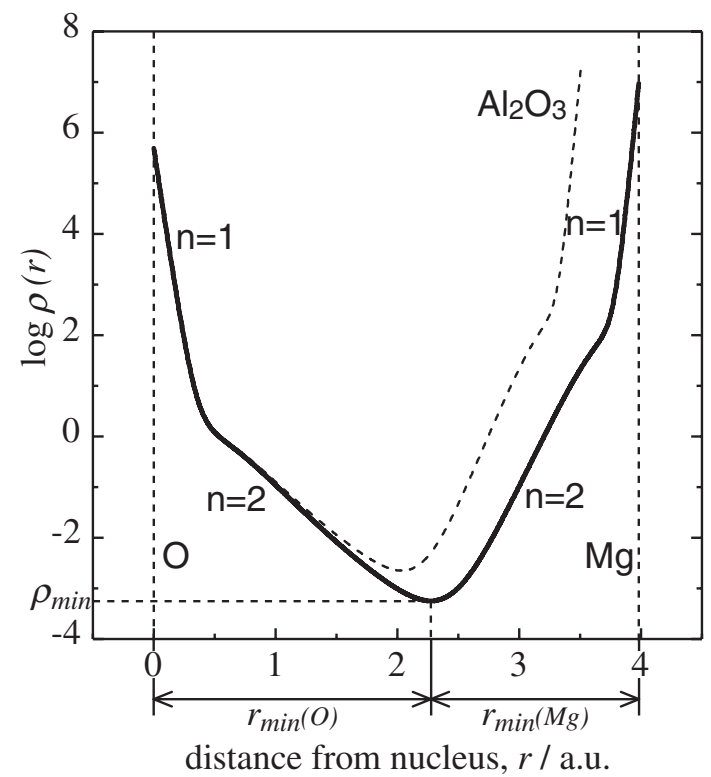

Fig. 2 Representation of natural logarithm of the electron density, $\log \rho(r)$, in the line linking the first-nearest neighbor $\mathrm{O}$ and $\mathrm{Mg}$ nuclei $(1 \mathrm{a} . \mathrm{u}$. $=$ $0.0529 \mathrm{~nm})$.Minimum electron density, $\rho_{\min }$, and ionic radii, $r_{\min }(O)$ for $\mathrm{O}$ and $r_{\min }(M g)$ for $\mathrm{Mg}$, are illustrated in the figure. A dotted curve is the result of $\mathrm{Al}_{2} \mathrm{O}_{3}$.

principal quantum number, $n$, which is a quantum number to determine the energy and the size of atom roughly. In case of the oxygen shown in Fig. 2, $n=1$ for the inner region and $n=2$ for the outer region. The slope was about 15.75 for the inner region composed mainly of $\mathrm{O} 1 \mathrm{~s}$ electrons, and about 6.61 for the outer region composed mainly of $\mathrm{O} 2 \mathrm{~s}, 2 \mathrm{p}$ electrons. It is known that $2 Z / n$ is the slope expected from the radial distribution function of hydrogen-like atom consisting of only one electron, where $Z$ is the atomic number. As $Z=8$ for oxygen, the $2 Z / n$ values are 16 for $n=1$ and 8 for $n=2$, each value being close to the respective values of oxygen described above. The similarity is especially great for $n=1$.

It was also found that the extent of the region $(n=2)$ for $\mathrm{O}-2 \mathrm{~s}, 2 \mathrm{p}$ electrons was dependent strongly on the neighboring atom in various oxides. For example, the result of $\log \rho(r)$ in $\mathrm{Al}_{2} \mathrm{O}_{3}$ is indicated by a dotted curve in Fig. 2. The region of $n=2$ in $\mathrm{Al}_{2} \mathrm{O}_{3}$ is rather limited compared to that in $\mathrm{MgO}$, but the slope still remains unchanged with the neighboring metal atom. Even the valence electrons such as O-2s, 2p keep their own slope, instead of spreading over the space in a random manner. In addition, the extent of $n=2$ region shows the range of influence of oxygen. So, its extension means that an effective number of electrons increases at the oxygen site, resulting in a large and negative ionicity of oxygen. In this sense, the extent of $n=2$ region is a measure of the ionicity as well as the ionic size.

\subsubsection{Non-transition metal oxides}

The results of $\log \rho(r)$ for the NaCl-type oxides are shown in Fig. 3. The slope of $n=2$ region in the oxygen side remains unchanged, but the slope in the metal side is dependent on the metal elements as well as the values of $n$. Also, the results of $\mathrm{MgO}, \mathrm{ZnO}$ and $\mathrm{Na}_{2} \mathrm{O}$ are compared with each other in Fig. 4. An oxygen ion is surrounded by 6 cations

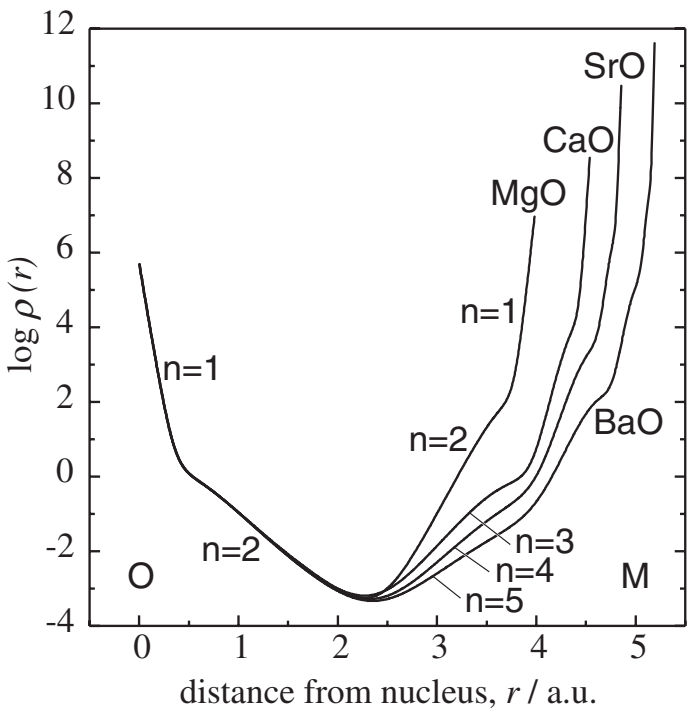

Fig. $3 \log \rho(r)$ vs. $r$ curves for the NaCl-type oxides, MO.

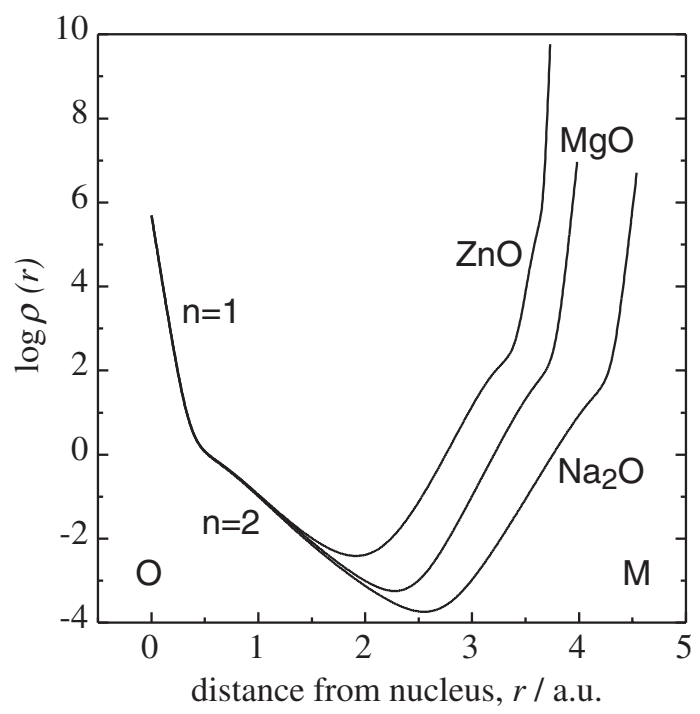

Fig. $4 \log \rho(r)$ vs. $r$ curves for the oxides, $\mathrm{MgO}, \mathrm{ZnO}$ and $\mathrm{Na}_{2} \mathrm{O}$.

in the first nearest neighbor in $\mathrm{MgO}$ with the $\mathrm{NaCl}$-type structure, and by 4 cations in $\mathrm{ZnO}$ with the wurtzite-type structure, and by 8 cations in $\mathrm{Na}_{2} \mathrm{O}$ with the $\mathrm{CaF}_{2}$-type structure. Despite these differences in the crystal structure and the coordination number among them, the slope of $n=2$ region in the oxygen side remains unchanged.

\subsubsection{Perovskite-type oxides}

Similar results are also seen in the perovskite-type oxides, $\mathrm{Sr}(\mathrm{B}) \mathrm{O}_{3}$, as shown in Fig. 5, where B's are Ti, Zr, Hf and $\mathrm{Ru}$. The slope in the metal side changes with the metal element, $\mathrm{B}$, but the slope in the oxygen side remains unchanged.

Furthermore, a $\log \rho(r)$ vs. $r$ plot for the perovskite-type oxide, $\mathrm{CaTiO}_{3}$, is shown in Fig. 6 together with that for the short Ti-O pair in the rutile-type oxide, $\mathrm{TiO}_{2}$. It is interesting that both the plots are coincident with each other, as long as the Ti-O interionic distance is the same between them. In other words, the effect of $\mathrm{Ca}$ is nearly null on the electron density along the Ti-O connecting line. This is probably due 


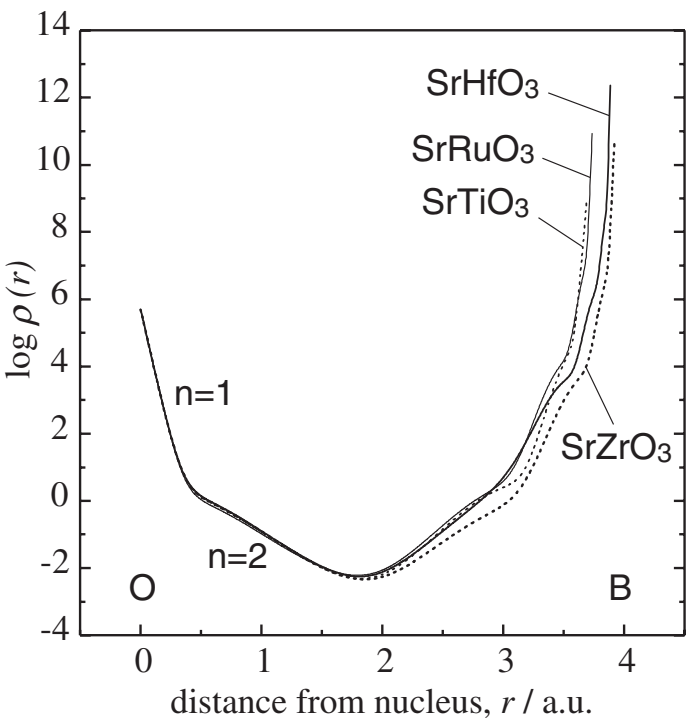

Fig. $5 \log \rho(r)$ vs. $r$ curves for the perovskite-type oxides, $\operatorname{Sr}(\mathrm{B}) \mathrm{O}_{3}$.

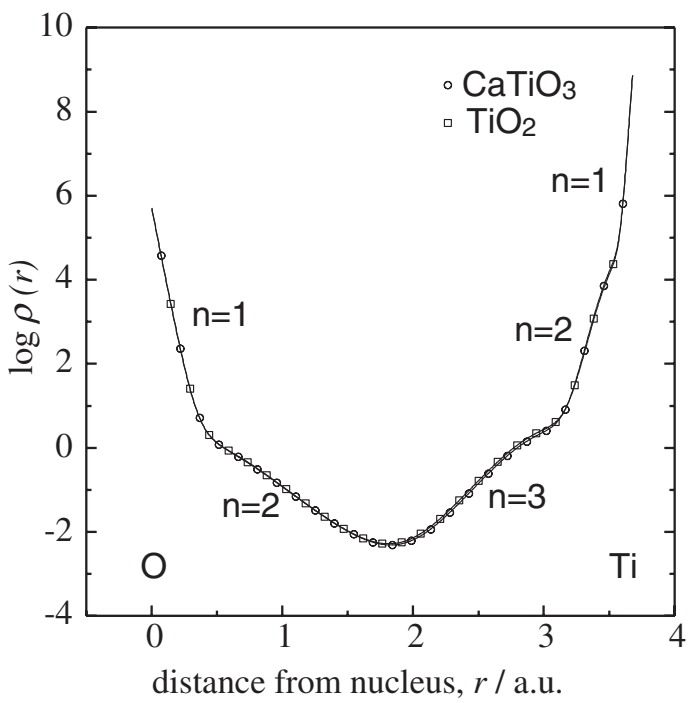

Fig. 6 Comparison of $\log \rho(r)$ vs. $r$ curves between $\mathrm{CaTiO}_{3}$ and $\mathrm{TiO}_{2}$. Here, Ti-O interionic distance was set to be same, $1.947 \mathrm{~nm}$, between them.

to the fact that most of $\mathrm{Ca}$ components are in the empty conduction band in $\mathrm{CaTiO}_{3}$. Thus, it was found that there are characteristic electron density distributions relevant to oxygen and metal elements in the oxides.

\subsection{Electron density distributions in borides, carbides, nitrides and fluorides}

\subsection{1 $\log \rho(r)$ vs. $r$ plots and their slopes}

A series of calculations was performed for other ceramics, and the $\log \rho(r)$ vs. $r$ plots are shown in Fig. 7(a) for borides, in Fig. 7(b) for carbides, in Fig. 7(c) for nitrides and in Fig. 7 (d) for fluorides.

In any cases each of $\mathrm{B}, \mathrm{C}, \mathrm{N}$ and $\mathrm{F}$ has own slopes, irrespective of the neighboring metal elements. Their slopes are summarized in Fig. 8 together with the results of O. For every element the slope of $n=1$ region is very close to the expected value from the radial distribution function of

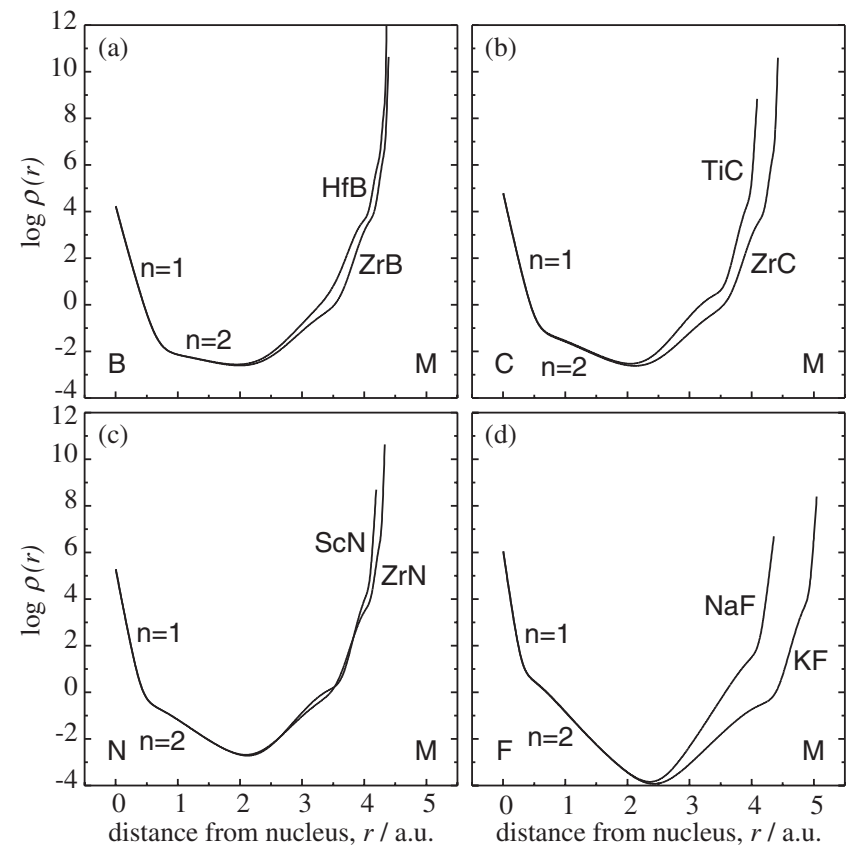

Fig. $7 \log \rho(r)$ vs. $r$ curves for (a) borides, (b) carbides, (c) nitrides and (d) fluorides.

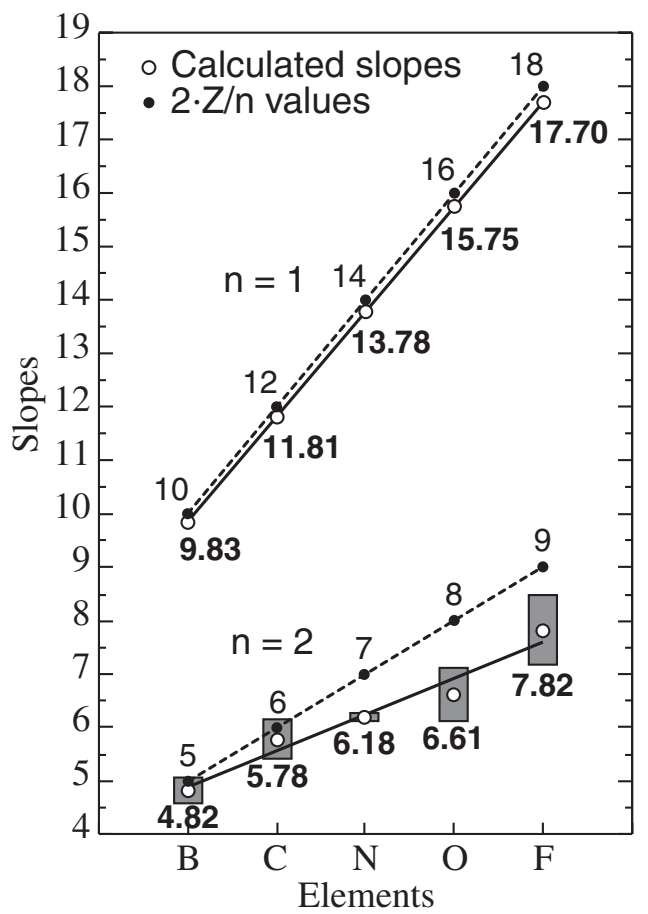

Fig. 8 Calculated slopes of elements, B, C, N, O and F, and comparison with the $2 Z / n$ values expected from the radial distribution function of hydrogen-like atom.

hydrogen-like atom. To confirm this further, the preexponential term, $\rho_{0}$, was calculated using an equation, $\rho(r)=\rho_{0} \exp (-\xi r)$ for $1 \mathrm{~s}$ electrons, where $\xi$ is the slope for $n=1$. According to the radial distribution function for $1 \mathrm{~s}$ electrons in the hydrogen-like atom, $\rho_{0}$ should be proportional to $Z^{3}$. This was indeed satisfied as shown in Fig. 9.

On the other hand, the slope of $n=2$ region has a trend of deviating slightly from the expected value as the atomic 


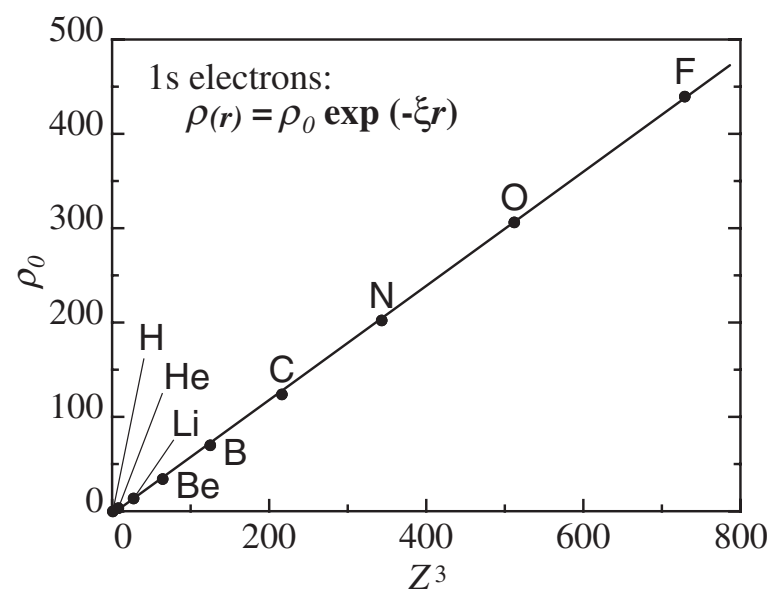

Fig. 9 Comparison between the calculated pre-exponential term, $\rho_{0}$, and the $Z^{3}$ term expected from the radial distribution function for $1 \mathrm{~s}$ electrons in hydrogen-like atom.

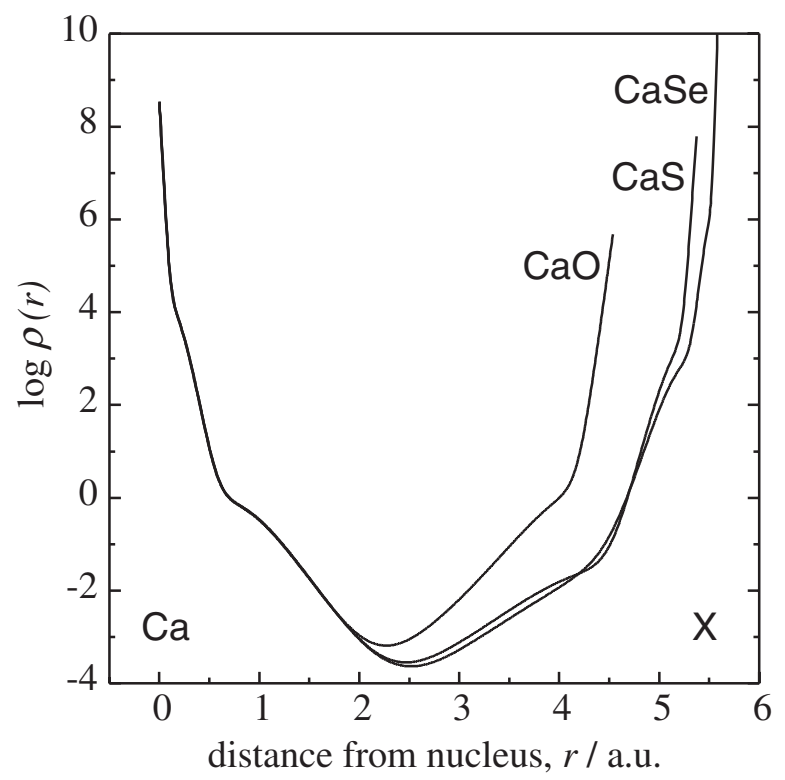

Fig. $10 \log \rho(r)$ vs. $r$ curves for the NaCl-type ceramics, $\mathrm{CaO}, \mathrm{CaS}$ and CaSe.

number increases, probably due to the increase in the screening of nuclear charges by a large number of electrons.

\subsubsection{Comparison of electron density distributions among ceramics}

In addition, to account for the difference among ceramics, $\mathrm{CaO}, \mathrm{CaS}$ and $\mathrm{CaSe}$ were examined, all of which have the $\mathrm{NaCl}$-type structure, and $\mathrm{O}, \mathrm{S}$ and $\mathrm{Se}$ are the 6B elements in the periodic table. The $\log \rho(r)$ vs. $r$ curves are shown in Fig. 10. In any curves the $n=4$ region in the $\mathrm{Ca}$ side is almost missing probably owing to the occurrence of the electron transfer from $\mathrm{Ca}$ to $\mathrm{O}$ or $\mathrm{S}$ or $\mathrm{Se}$, because ionic bonding characters are strong in these ceramics. The extent of the outer region in the $\mathrm{Ca}$ side is smaller in $\mathrm{CaO}$ than $\mathrm{CaS}$ and $\mathrm{CaSe}$, so that the effective positive charge on the Ca site must be larger in $\mathrm{CaO}$ than $\mathrm{CaS}$ and $\mathrm{CaSe}$. In other words, ionic bonding characters are probably stronger in $\mathrm{CaO}$ than $\mathrm{CaS}$ and CaSe.

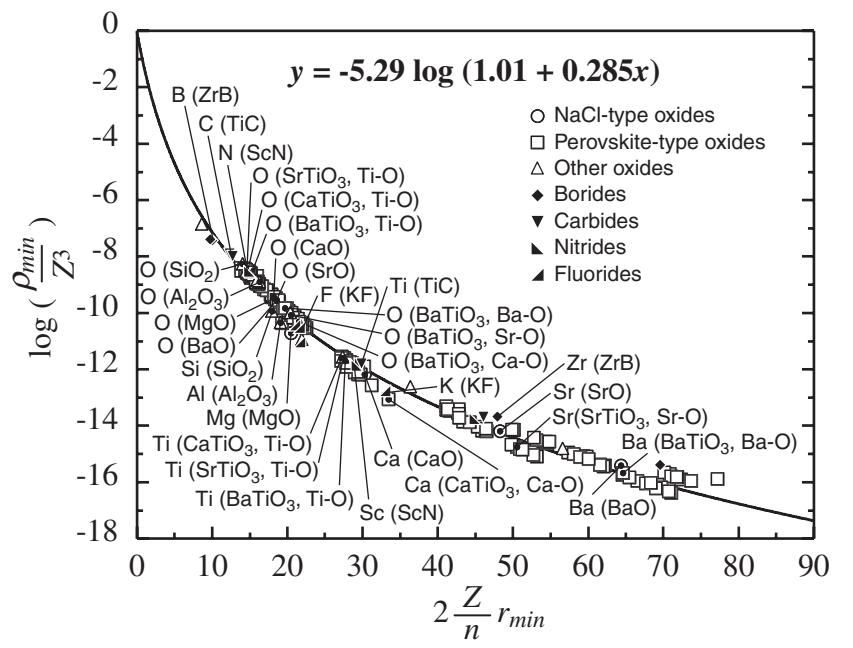

Fig. 11 A universal relation of several oxides and bolides, carbides, nitrides and fluorides between the minimum electron density, $\rho_{\min }$, and the atomic or ionic radii, $r_{\min }$. Notations used in the figure; for example, Ti(or $\mathrm{O})\left(\mathrm{BaTiO}_{3}, \mathrm{Ti}-\mathrm{O}\right)$ denotes $\mathrm{Ti}($ or $\mathrm{O})$ in the first-nearest neighbor Ti-O pair in $\mathrm{BaTiO}_{3}$. $\mathrm{Ti}(\mathrm{TiC})$ and $\mathrm{C}(\mathrm{TiC})$ denote $\mathrm{Ti}$ and $\mathrm{C}$ in the first-nearest neighbor Ti-C pair in TiC, respectively. The others are denoted in a similar way.

\subsection{A universal curve between electron density minima and ionic radii}

Next, as illustrated in Fig. 2, we defined the minimum electron density, $\rho_{\mathrm{min}}$, and the ionic radii, $r_{\mathrm{min}}$, at the position of showing $\rho_{\min }$. The values of $\rho_{\min }$ and $r_{\min }$ were calculated for various oxides of over 50 species, and the results are summarized in Fig. 11. In this figure the vertical axis is $\log \left(\rho_{\min } / Z^{3}\right)$ and the horizontal axis is $2(Z / n) r_{\min }$. This coordinate was chosen, because in case of the radial distribution functions of hydrogen-like atom, $\rho(r) / Z^{3}$ is a function of only one variable, $2(Z / n) r$.

It is surprising that every oxides falls on one curve, despite the difference in the crystal structures among them. For example, some of the oxides named in Fig. 11 are the $\mathrm{NaCl}-$ type oxides $(\mathrm{MgO}, \mathrm{CaO}, \mathrm{SrO}, \mathrm{BaO})$, the perovskite-type oxides $\left(\mathrm{BaTiO}_{3}, \mathrm{SrTiO}_{3}, \mathrm{CaTiO}_{3}\right)$ and other oxides $\left(\mathrm{Al}_{2} \mathrm{O}_{3}\right.$, $\mathrm{SiO}_{2}$ ). Not only oxides but also other ceramics also fall on this curve as shown in Fig. 11. Thus, this curve is indeed a universal curve common to every ceramic material. But this universal curve is satisfied not only in ceramic materials but also in other materials including metals, semiconductors, ionic crystals and gas molecules. ${ }^{8}$ )

By the least-squared analysis, a universal curve is expressed in a simple formula as,

$$
\log \left[\rho_{\min } / Z^{3}\right]=a \cdot \log \left[b+c \cdot 2(Z / n) r_{\min }\right] .
$$

Each of the fitting parameters was, $a=-5.29 \pm 0.066, b=$ $1.01 \pm 0.040$ and $c=0.285 \pm 0.010$.

The eq. (1) is useful, for example, for the determination of ionic radii. Let's explain this using an $\mathrm{AB}$ binary ceramic with the first-nearest-neighbor $\mathrm{A}-\mathrm{B}$ distance, $r_{0}$. If $2(Z /$ $n) r_{\min }=2\left(Z_{\mathrm{A}} / n_{\mathrm{A}}\right) r_{\mathrm{A}}$ for $\mathrm{A}$ and $2(Z / n) r_{\min }=2\left(Z_{\mathrm{B}} / n_{\mathrm{B}}\right) r_{\mathrm{B}}$ for $\mathrm{B}$, then $r_{\mathrm{A}}+r_{\mathrm{B}}=r_{0}$. Also, $\rho_{\min }$ is the minimum electron density at the $r_{\mathrm{A}}$ position from $\mathrm{A}$ nucleus and at the $r_{\mathrm{B}}$ position from B nucleus in the A-B connecting line (see Fig. 2 
for the case of $\mathrm{MgO}$ ). Under this condition, the following linear equation between $r_{\mathrm{A}}$ and $r_{\mathrm{B}}$ is derived by manipulating the eq. (1),

$$
\begin{aligned}
& 2\left(Z_{\mathrm{A}} / n_{\mathrm{A}}\right) r_{\mathrm{A}}-\alpha \cdot 2\left(Z_{\mathrm{B}} / n_{\mathrm{B}}\right) r_{\mathrm{B}}=(b / c) \cdot(\alpha-1), \\
& \alpha=\exp \left[(1 / a) \log \left(Z_{\mathrm{B}}^{3} / Z_{\mathrm{A}}^{3}\right)\right] .
\end{aligned}
$$

As $r_{\mathrm{A}}+r_{\mathrm{B}}=r_{0}, r_{\mathrm{A}}$ and $r_{\mathrm{B}}$ are obtained by solving these simultaneous equations. The results are expressed as,

$$
\begin{gathered}
r_{\mathrm{A}}=\left[r_{0} \cdot \alpha \cdot 2\left(Z_{\mathrm{B}} / n_{\mathrm{B}}\right)+(b / c) \cdot(\alpha-1)\right] / \beta, \\
r_{\mathrm{B}}=\left[r_{0} \cdot 2\left(Z_{\mathrm{A}} / n_{\mathrm{A}}\right)-(b / c) \cdot(\alpha-1)\right] / \beta, \\
\beta=2\left(Z_{\mathrm{A}} / n_{\mathrm{A}}\right)+\alpha \cdot 2\left(Z_{\mathrm{B}} / n_{\mathrm{B}}\right) .
\end{gathered}
$$

Thus, the ionic radii, $r_{\mathrm{A}}$ and $r_{\mathrm{B}}$, are calculated from a given $r_{0}$, using the eqs. $(3 \mathrm{a}, 3 \mathrm{~b})$. Then, $\rho_{\min }$ is calculated from the eq. (1). In most materials $\rho_{\min }$ correlates with the cohesive energy, ${ }^{9)}$ so its prediction is useful for materials design. Also, the ionic radii have been proposed by many investigators, ${ }^{10}$ ) but strictly speaking they are not able to be defined correctly, since the ionic radius, $r_{\mathrm{A}}$, is dependent on the neighboring $\mathrm{B}$ ion, as shown in the eq. (3a). Further study is now underway to account for the physical meaning of the coefficients, $a, b$, and $c$, in the eq. (1).

\section{Conclusion}

From first principles calculations of electronic structures in ceramics over 50 species, a universal relation was discovered between electron density minima and ionic radii. Using this relation, ionic radii and the minimum electron density in any ceramics can be calculated when the first-nearest-neighbor distance is known.

\section{Acknowledgments}

The authors would like to express sincere thanks to the staffs of the Computer Center, Institute for Molecular Science, Okazaki National Institute for the use of supercomputers. This study was supported by a Grant-in-Aid for Scientific Research from the Ministry of Education, Culture, Sports, Science and Technology of Japan, and also by the Japan Society for the Promotion of Science.

\section{REFERENCES}

1) R. S. Mulliken: J. Chem. Phys. 23 (1955) 1841-1849.

2) R. S. Mulliken: J. Chem. Phys. 23 (1955) 2338-2342.

3) R. F. W. Bader and H. Essen: J Chem. Phys. 80 (1984) 1943-1960.

4) J. C. Slater: The Calculation of Molecular Orbitals, (Wiley, New York, 1979).

5) F. W. Averill and D. E. Ellis: J. Chem. Phys. 59 (1973) 6412-6418.

6) H. Adachi, M. Tsukada and C. Satoko: J. Phys. Soc. Jpn. 45 (1978) $875-883$.

7) M. Morinaga, N. Yukawa and H. Adachi: J. Phys. Soc. Jpn. 53 (1984) $653-663$.

8) M. Morinaga and M. Yoshino: to be published.

9) Y. Aray, J. Rodriguez and D. Vega: J. Phys. Chem. B 104 (2000) 46084612.

10) R. D. Shannon: Acta Crystallogr. A32 (1976) 751-767. 